\title{
An analogue of Gutzmer's formula for Hermite expansions
}

\author{
by
}

\section{S. Thangavelu (Bangalore)}

Dedicated to Somesh Bagchi on his sixtieth birthday

\begin{abstract}
We prove an analogue of Gutzmer's formula for Hermite expansions. As a consequence we obtain a new proof of a characterisation of the image of $L^{2}\left(\mathbb{R}^{n}\right)$ under the Hermite semigroup. We also obtain some new orthogonality relations for complexified Hermite functions.
\end{abstract}

1. Introduction. By Gutzmer's formula we mean any analogue of the formula

$$
(2 \pi)^{-1} \int_{0}^{2 \pi}|f(x+i y)|^{2} d x=\sum_{k=-\infty}^{\infty}|\widehat{f}(k)|^{2} e^{-2 k y}
$$

valid for any $2 \pi$-periodic holomorphic function $f$ in a strip in the complex plane. Here $\widehat{f}(k)$ stands for the Fourier coefficients of the restriction of $f$ to the real line. An analogue of such a formula was established by Lassalle [9] for holomorphic functions on the complexifications of compact symmetric spaces. A similar formula for holomorphic functions on the complex crowns associated to noncompact Riemannian symmetric spaces was discovered by Faraut [3]. As can be seen from Faraut [2] and Krötz-Ólafsson-Stanton [7] such formulas are useful in the study of Segal-Bargmann or heat kernel transforms.

Recently in [15] we have proved an analogue of Gutzmer's formula on the Heisenberg groups and used them to study heat kernel transforms and Paley-Wiener theorems.

In this paper we prove an analogue of Gutzmer's formula for Hermite expansions. Let $H$ be the Hermite operator on $\mathbb{R}^{n}$ having the spectral decomposition $H=\sum_{k=0}^{\infty}(2 k+n) P_{k}$. Let $\mathbb{H}^{n}=\mathbb{R}^{n} \times \mathbb{R}^{n} \times \mathbb{R}$ be the Heisenberg group whose complexification is $\mathbb{C}^{n} \times \mathbb{C}^{n} \times \mathbb{C}$. Let $\pi(x, u)$ be the pro-

2000 Mathematics Subject Classification: Primary 42A38; Secondary 42B99, 43A90.

Key words and phrases: Hermite and Laguerre functions, Heisenberg group, symplectic, orthogonal and unitary matrices, entire functions. 
jective representation of $\mathbb{R}^{n} \times \mathbb{R}^{n}$ related to the Schrödinger representation of $\mathbb{H}^{n}$ and denote by $\pi(x+i y, u+i v)$ its extension to $\mathbb{C}^{n} \times \mathbb{C}^{n}$. Let $K=\operatorname{Sp}(n, \mathbb{R}) \cap O(2 n, \mathbb{R})$, which acts on $\mathbb{C}^{n} \times \mathbb{C}^{n}$. Denote by $\varphi_{k}(z, w)$ the Laguerre functions of type $(n-1)$ extended to $\mathbb{C}^{n} \times \mathbb{C}^{n}$. Our main result is the following.

Theorem 1.1. Let $F$ be an entire function on $\mathbb{C}^{n}$. Denote by $f$ its restriction to $\mathbb{R}^{n}$. Then for any $z=x+i y, w=u+i v \in \mathbb{C}^{n}$ we have

$$
\begin{aligned}
\int_{\mathbb{R}^{n}} \int_{K}|\pi(\sigma \cdot(z, w)) F(\xi)|^{2} d \sigma d \xi & \\
& =e^{(u \cdot y-v \cdot x)} \sum_{k=0}^{\infty} \frac{k !(n-1) !}{(k+n-1) !} \varphi_{k}(2 i y, 2 i v)\left\|P_{k} f\right\|_{2}^{2} .
\end{aligned}
$$

As an immediate corollary we obtain the following characterisation of the image of $L^{2}\left(\mathbb{R}^{n}\right)$ under the Hermite semigroup $e^{-t H}, t>0$. Let

$$
U_{t}(x, y)=2^{n}(\sinh (4 t))^{-n / 2} e^{\tanh (2 t)|x|^{2}-\operatorname{coth}(2 t)|y|^{2}} .
$$

Corollary 1.2. An entire function $F$ on $\mathbb{C}^{n}$ belongs to the image of $L^{2}\left(\mathbb{R}^{n}\right)$ under $e^{-t H}$ if and only if

$$
\int_{\mathbb{R}^{n}} \int_{\mathbb{R}^{n}}|F(x+i y)|^{2} U_{t}(x, y) d x d y<\infty .
$$

This characterisation is not new and there are several proofs available in the literature (see Byun [1], Karp [6] and Thangavelu [14]). In Section 4 we derive some more consequences of Gutzmer's formula.

We conclude this introduction with some remarks about the methods used in proving Gutzmer's formulas. As in the case of Fourier series, Lassalle [9] used Plancherel's theorem for the Laurent expansions of holomorphic functions on the complexifications of compact symmetric spaces $X=K / M$. The matrix coefficients associated to class one representations in the unitary dual of a compact Lie group $K$ holomorphically extend to its complexification $K_{\mathbb{C}}$. Thus any function $f$ whose "Fourier coefficients" have exponential decay can be extended to the complexification $X_{\mathbb{C}}=K_{\mathbb{C}} / M_{\mathbb{C}}$. Then by appealing to Plancherel's theorem and using orthogonality relations the required formula was established. In [2] Faraut considered a general unimodular group $G$ and proved a proposition from which Gutzmer's formula can be deduced for noncompact Riemannian symmetric spaces [3] and Heisenberg groups [15].

Thus in all the previous settings the basic functions appearing in the Fourier series or transform are matrix coefficients of certain irreducible unitary representations of the underlying group. In contrast, the Hermite functions do not occur as matrix coefficients. However, the Hermite functions are used to calculate the matrix coefficients associated to Schrödinger rep- 
resentations of $\mathbb{H}^{n}$ resulting in special Hermite or Laguerre functions. This explains why the representation $\pi(z, w)$ occurs in our Gutzmer's formula. The close relationship between Hermite and Laguerre functions is then used to derive Gutzmer's formula.

2. Preliminaries. In this section we collect some relevant information about special Hermite functions and prove some results that are required in the next section. We closely follow the notations used in [12] and [13] and we refer the reader to these monographs for more details.

Let $\Phi_{\alpha}, \alpha \in \mathbb{N}^{n}$, be the Hermite functions on $\mathbb{R}^{n}$ normalised so that their $L^{2}$ norms are one. These are eigenfunctions of the Hermite operator $H=-\Delta+|x|^{2}$ with eigenvalues $2|\alpha|+n$. On finite linear combinations of such functions we can define certain operators $\pi(z, w)$, where $z, w \in \mathbb{C}^{n}$, as follows:

$$
\pi(z, w) \Phi_{\alpha}(\xi)=e^{i\left(z \cdot \xi+\frac{1}{2} z \cdot w\right)} \Phi_{\alpha}(\xi+w)
$$

where $z \cdot \xi=\sum_{j=1}^{n} z_{j} \xi_{j}$ and $z \cdot w=\sum_{j=1}^{n} z_{j} w_{j}$. Note that $\Phi_{\alpha}(\xi)=H_{\alpha}(\xi) e^{-\frac{1}{2}|\xi|^{2}}$ where $H_{\alpha}$ is a polynomial on $\mathbb{R}^{n}$ and for $z \in \mathbb{C}^{n}$ we define $\Phi_{\alpha}(z)$ to be $H_{\alpha}(z) e^{-\frac{1}{2} z^{2}}$, where $z^{2}=z \cdot z$. The special Hermite functions $\Phi_{\alpha, \beta}(z, w)$ are then defined by

$$
\Phi_{\alpha, \beta}(z, w)=(2 \pi)^{-n / 2}\left(\pi(z, w) \Phi_{\alpha}, \Phi_{\beta}\right) .
$$

The restrictions of $\Phi_{\alpha, \beta}(z, w)$ to $\mathbb{R}^{n} \times \mathbb{R}^{n}$ are usually called the special Hermite functions and the family $\left\{\Phi_{\alpha, \beta}(x, u): \alpha, \beta \in \mathbb{N}^{n}\right\}$ forms an orthonormal basis for $L^{2}\left(\mathbb{C}^{n}\right)$ (see Proposition 1.4.1 in [12]).

As we have mentioned in the introduction, the operators $\pi(z, w)$ are related to the Schrödinger representation $\pi_{1}$ of the Heisenberg group $\mathbb{H}^{n}$. Recall that $\mathbb{H}^{n}=\mathbb{R}^{n} \times \mathbb{R}^{n} \times \mathbb{R}$ is equipped with the group law $(x, u, t)\left(x^{\prime}, u^{\prime}, t^{\prime}\right)=$ $\left(x+x^{\prime}, u+u^{\prime}, t+t^{\prime}+\frac{1}{2}\left(u \cdot x^{\prime}-x \cdot u^{\prime}\right)\right)$. For each nonzero real number $\lambda$ we have a representation of $\mathbb{H}^{n}$ realised on $L^{2}\left(\mathbb{R}^{n}\right)$ given by

$$
\pi_{\lambda}(x, u, t) \varphi(\xi)=e^{i \lambda t} e^{i \lambda\left(x \cdot \xi+\frac{1}{2} x \cdot u\right)} \varphi(\xi+u) .
$$

Thus $\pi(x, u)=\pi_{1}(x, u, 0)$ defines a projective representation of $\mathbb{R}^{n} \times \mathbb{R}^{n}$.

For $(z, w) \in \mathbb{C}^{2 n}$ the operators $\pi(z, w)$ are not even bounded on $L^{2}\left(\mathbb{R}^{n}\right)$. However, they are densely defined and satisfy

$$
\pi(z, w) \pi\left(z^{\prime}, w^{\prime}\right)=e^{(i / 2)\left(z^{\prime} \cdot w-z \cdot w^{\prime}\right)} \pi\left(z+z^{\prime}, w+w^{\prime}\right) .
$$

Moreover,

$$
\left(\pi(i y, i v) \Phi_{\alpha}, \Phi_{\beta}\right)=\left(\Phi_{\alpha}, \pi(i y, i v) \Phi_{\beta}\right) .
$$

This means that $\pi(i y, i v)$ are self-adjoint operators. We need to calculate the $L^{2}$ norms of $\pi(z, w) \Phi_{\alpha}$. Let $L_{k}^{n-1}$ be Laguerre polynomials of type $(n-1)$ and define the Laguerre functions $\varphi_{k}$ by

$$
\varphi_{k}(x, u)=L_{k}^{n-1}\left(\frac{1}{2}\left(x^{2}+u^{2}\right)\right) e^{-\frac{1}{4}\left(x^{2}+u^{2}\right)} .
$$


Then it is known (see Section 1.4 in [12]) that

$$
\varphi_{k}(x, u)=(2 \pi)^{n / 2} \sum_{|\alpha|=k} \Phi_{\alpha, \alpha}(x, u) .
$$

These functions have a natural holomorphic extension to $\mathbb{C}^{n} \times \mathbb{C}^{n}$, denoted by the same symbol:

$$
\varphi_{k}(z, w)=(2 \pi)^{n / 2} \sum_{|\alpha|=k} \Phi_{\alpha, \alpha}(z, w) .
$$

Lemma 2.1. For any $z=x+i y, w=u+i v \in \mathbb{C}^{n}$ and $\alpha \in \mathbb{N}^{n}$ we have

$$
\int_{\mathbb{R}^{n}}\left|\pi(z, w) \Phi_{\alpha}(\xi)\right|^{2} d \xi=(2 \pi)^{n / 2} e^{u \cdot y-v \cdot x} \Phi_{\alpha, \alpha}(2 i y, 2 i v) .
$$

Proof. It is enough to prove the result in one dimension. Recall Mehler's formula satisfied by the Hermite functions $h_{k}$ on $\mathbb{R}$ :

$$
\sum_{k=0}^{\infty} h_{k}(\xi) h_{k}(\eta) r^{k}=\pi^{-1 / 2}\left(1-r^{2}\right)^{-1 / 2} e^{-\frac{1}{2} \frac{1+r^{2}}{1-r^{2}}\left(\xi^{2}+\eta^{2}\right)+\frac{2 r}{1-r^{2}} \xi \eta},
$$

valid for all $r$ with $|r|<1$. The formula is clearly valid even if $\xi$ and $\eta$ are complex. A simple calculation shows that

$$
\sum_{k=0}^{\infty} r^{k}\left|\pi(z, w) h_{k}(\xi)\right|^{2}=\pi^{-1 / 2}\left(1-r^{2}\right)^{-1 / 2} e^{-(u y+v x)} e^{\frac{1+r}{1-r} v^{2}} e^{-\frac{1-r}{1+r}(\xi+u)^{2}} e^{-2 y \xi} .
$$

Integrating both sides with respect to $\xi$ we obtain

$$
\sum_{k=0}^{\infty} r^{k} \int_{\mathbb{R}}\left|\pi(z, w) h_{k}(\xi)\right|^{2} d \xi=(1-r)^{-1} e^{u y-v x} e^{\frac{1+r}{1-r}\left(y^{2}+v^{2}\right)}
$$

We now recall that the generating function for the Laguerre functions $\varphi_{k}(x, u)$ when $n=1$ is

$$
\sum_{k=0}^{\infty} r^{k} \varphi_{k}(x, u)=(1-r)^{-1} e^{-\frac{1}{4} \frac{1+r}{1-r}\left(x^{2}+u^{2}\right)} .
$$

A comparison with this shows that

$$
\int_{\mathbb{R}}\left|\pi(z, w) h_{k}(\xi)\right|^{2} d \xi=e^{u y-v x} \varphi_{k}(2 i y, 2 i v) .
$$

Since $\Phi_{k, k}(x, u)=(2 \pi)^{-1 / 2} \varphi_{k}(x, u)$, this proves the lemma.

In the above lemma we have calculated the $L^{2}$ norm of $\pi(z, w) \Phi_{\alpha}$ by integrating the generating function. We can also calculate the norm by expanding $\pi(z, w) \Phi_{\alpha}$ in terms of the Hermite basis and appealing to the Plancherel theorem for Hermite expansions. This leads to the following identity which is crucial for our main result. 
Lemma 2.2. For any $\alpha \in \mathbb{N}^{n}, z=x+i y, w=u+i v \in \mathbb{C}^{n}$ we have

$$
\sum_{\beta \in \mathbb{N}^{n}}\left|\Phi_{\alpha, \beta}(z, w)\right|^{2}=(2 \pi)^{-n / 2} e^{u \cdot y-v \cdot x} \Phi_{\alpha, \alpha}(2 i y, 2 i v) .
$$

Proof. We just have to recall that

$$
\left(\pi(z, w) \Phi_{\alpha}, \Phi_{\beta}\right)=(2 \pi)^{n / 2} \Phi_{\alpha, \beta}(z, w) .
$$

We also need some estimates on the holomorphically extended Hermite functions on $\mathbb{C}^{n}$. Let us define $\Phi_{k}(x, u)=\sum_{|\alpha|=k} \Phi_{\alpha}(x) \Phi_{\alpha}(u)$, which is the kernel of the projection $P_{k}$. Note that $\Phi_{k}$ extends to $\mathbb{C}^{n} \times \mathbb{C}^{n}$ as an entire function. Using Mehler's formula for Hermite functions and the generating function for Laguerre functions we can get the following representation of $\Phi_{k}$ in terms of Laguerre functions of type $(n / 2-1)$.

LEMMA 2.3.

$\Phi_{k}(z, w)=\pi^{-n / 2} \sum_{j=0}^{k}(-1)^{j} L_{j}^{n / 2-1}\left(\frac{1}{2}(z+w)^{2}\right) L_{k-j}^{n / 2-1}\left(\frac{1}{2}(z-w)^{2}\right) e^{-\frac{1}{2}\left(z^{2}+w^{2}\right)}$, where $z^{2}=\sum_{j=1}^{n} z_{j}^{2}$ and $w^{2}=\sum_{j=1}^{n} w_{j}^{2}$.

Proof. The Laguerre functions of type $(n / 2-1)$ are given by the generating function

$$
\sum_{k=0} r^{k} L_{k}^{n / 2-1}\left(\frac{1}{2} z^{2}\right) e^{-\frac{1}{4} z^{2}}=(1-r)^{-n / 2} e^{-\frac{1}{4} \frac{1+r}{1-r} z^{2}}
$$

A simple calculation shows that

$$
\begin{aligned}
(1-r)^{-n / 2} e^{-\frac{1}{4} \frac{1+r}{1-r}(z+w)^{2}}(1+r)^{-n / 2} e^{-\frac{1}{4} \frac{1-r}{1+r}(z-w)^{2}} & \\
& =\left(1-r^{2}\right)^{-n / 2} e^{-\frac{1}{2} \frac{1+r^{2}}{1-r^{2}}\left(z^{2}+w^{2}\right)+\frac{2 r}{1-r^{2}} z w} .
\end{aligned}
$$

Comparing this with Mehler's formula and rewriting the left hand side as a power series in $r$ and then equating coefficients of $r^{k}$ we obtain the lemma.

The above lemma has already been used by us in the study of BochnerRiesz means for multiple Hermite expansions. Here we need the above in order to get the following estimate on $\Phi_{k}(z, w)$.

Lemma 2.4. For all $z=x+i y \in \mathbb{C}^{n}$ and $k=1,2, \ldots$ we have

$$
\left|\Phi_{k}(z, \bar{z})\right| \leq C(y) k^{\frac{3}{4}(n-1)} e^{2 k^{1 / 2}|y|},
$$

where $C(y)$ is locally bounded.

Proof. From the previous lemma we have

$$
\Phi_{k}(z, \bar{z})=\pi^{-n / 2} \sum_{j=0}^{k}(-1)^{j} L_{j}^{n / 2-1}\left(2|x|^{2}\right) e^{-|x|^{2}} L_{k-j}^{n / 2-1}\left(-2|y|^{2}\right) e^{|y|^{2}} .
$$


We now make use of the following estimates on Laguerre functions. First of all we know that

$$
\left|L_{j}^{n / 2-1}\left(2|x|^{2}\right) e^{-|x|^{2}}\right| \leq C j^{n / 2-1}
$$

uniformly in $x$. On the other hand, Perron's formula for Laguerre polynomials in the complex domain (see Theorem 8.22.3 in Szegö [11]) gives us

$$
L_{j}^{n / 2-1}\left(-2|y|^{2}\right) e^{|y|^{2}} \leq C(y) j^{(n-3) / 4} e^{2 j^{1 / 2}|y|},
$$

valid for all $|y| \geq 1$. Since $L_{j}^{n / 2-1}\left(-2|y|^{2}\right) \leq L_{j}^{n / 2-1}(-2)$ for $|y| \leq 1$ we have the same estimate for all values of $y$. These two estimates imply the required bound on $\Phi_{k}(z, \bar{z})$.

We conclude the preliminaries with establishing some more notation. Let $\operatorname{Sp}(n, \mathbb{R})$ stand for the symplectic group consisting of $2 n \times 2 n$ real matrices that preserve the symplectic form $[(x, u),(y, v)]=u \cdot y-v \cdot x$ on $\mathbb{R}^{2 n}$ and have determinant one. Let $O(2 n, \mathbb{R})$ be the orthogonal group; we define $K=\operatorname{Sp}(n, \mathbb{R}) \cap O(2 n, \mathbb{R})$. Then there is a one-to-one correspondence between $K$ and the unitary group $U(n)$. Let $\sigma=a+i b$ be an $n \times n$ complex matrix with real and imaginary parts $a$ and $b$. Then $\sigma$ is unitary if and only if the matrix $A=\left(\begin{array}{cc}a & -b \\ b & a\end{array}\right)$ is in $K$. For these facts we refer to Folland [4]. By $\sigma .(x, u)$ we denote the action of the correspoding matrix $A$ on $(x, u)$. This action has a natural extension to $\mathbb{C}^{n} \times \mathbb{C}^{n}$ denoted by $\sigma \cdot(z, w)$ and given by $\sigma .(z, w)=(a . z-b . w, a . w+b . z)$ where $\sigma=a+i b$ as above. For example, when $n=1$ and $\sigma=e^{i \theta}$ we see that the corresponding matrix $A$ is $\left(\begin{array}{c}\cos \theta-\sin \theta \\ \sin \theta \\ \cos \theta\end{array}\right)$. Given $\theta=\left(\theta_{1}, \ldots, \theta_{n}\right) \in \mathbb{R}^{n}$ we denote by $k(\theta)$ the diagonal matrix in $U(n)$ with entries $e^{i \theta_{j}}$. We denote by $d \sigma$ the normalised Haar measure on $K$ and by $d \theta$ the Lebesgue measure $d \theta_{1} \cdots d \theta_{n}$.

3. The main results. Having set up notation and collected relevant results on special Hermite functions we are now ready to prove our main results. We begin with

THEOREM 3.1. Let $f \in L^{2}\left(\mathbb{R}^{n}\right)$ be such that $\left\|P_{k} f\right\|_{2} \leq C_{t} e^{-2 k^{1 / 2} t}$ for all $t>0$ and $k \in \mathbb{N}$. Then $f$ has a holomorphic extension $F$ to $\mathbb{C}^{n}$ and we have the following formula for any $z=x+i y, w=u+i v \in \mathbb{C}^{n}$ :

$$
\int_{\mathbb{R}^{n}} \int_{K}|\pi(\sigma \cdot(z, w)) F(\xi)|^{2} d \sigma d \xi=e^{u \cdot y-v \cdot x} \sum_{k=0}^{\infty} \frac{k !(n-1) !}{(k+n-1) !} \varphi_{k}(2 i y, 2 i v)\left\|P_{k} f\right\|_{2}^{2} .
$$

Proof. Consider the Hermite expansion of the function $f$ given by

$$
f(x)=\sum_{k=0}^{\infty} \sum_{|\alpha|=k}\left(f, \Phi_{\alpha}\right) \Phi_{\alpha}(x)
$$


By the Cauchy-Schwarz inequality,

$$
\left|\sum_{|\alpha|=k}\left(f, \Phi_{\alpha}\right) \Phi_{\alpha}(x+i y)\right|^{2} \leq \Phi_{k}(x+i y, x-i y)\left\|P_{k} f\right\|_{2}^{2} .
$$

In view of Lemma 2.4 the hypothesis on $f$ allows us to conclude that the series

$$
\sum_{k=0}^{\infty} \sum_{|\alpha|=k}\left(f, \Phi_{\alpha}\right) \Phi_{\alpha}(x+i y)
$$

converges uniformly over compact subsets of $\mathbb{C}^{n}$ and hence $f$ extends to an entire function $F$ on $\mathbb{C}^{n}$.

Let $D$ be the subgroup of $K$ consisting of $2 n \times 2 n$ matrices associated to the elements $k(\theta) \in U(n)$. We claim that it is enough to prove

$$
\begin{aligned}
&(2 \pi)^{-n} \int_{\mathbb{R}^{n}} \int_{D}|\pi(k(\theta) \cdot(z, w)) F(\xi)|^{2} d \theta d \xi \\
&=(2 \pi)^{n / 2} e^{u \cdot y-v \cdot x} \sum_{\alpha \in \mathbb{N}^{n}} \Phi_{\alpha, \alpha}(2 i y, 2 i v)\left|\left(f, \Phi_{\alpha}\right)\right|^{2} .
\end{aligned}
$$

To see the claim, suppose we have the above formula. Then writing

$$
\begin{array}{rl}
\int_{\mathbb{R}^{n}} \int_{K}|\pi(\sigma \cdot(z, w)) F(\xi)|^{2} & d \sigma d \xi \\
& =(2 \pi)^{-n} \int_{\mathbb{R}^{n}} \int_{D} \int_{K}|\pi(k(\theta) \sigma \cdot(z, w)) F(\xi)|^{2} d \sigma d \theta d \xi
\end{array}
$$

we get

$$
\begin{aligned}
& \int_{\mathbb{R}^{n}} \int_{K}|\pi(\sigma \cdot(z, w)) F(\xi)|^{2} d \sigma d \xi \\
&=(2 \pi)^{n / 2} \int_{K} e^{u^{\prime} \cdot y^{\prime}-v^{\prime} \cdot x^{\prime}} \sum_{\alpha \in \mathbb{N}^{n}} \Phi_{\alpha, \alpha}\left(2 i y^{\prime}, 2 i v^{\prime}\right)\left|\left(f, \Phi_{\alpha}\right)\right|^{2} d \sigma,
\end{aligned}
$$

where $\left(z^{\prime}, w^{\prime}\right)=\sigma \cdot(z, w)$. Since the action of $\sigma$ preserves the symplectic form we have $e^{u \cdot y-v \cdot x}=e^{u^{\prime} \cdot y^{\prime}-v^{\prime} \cdot x^{\prime}}$. Thus we are left with proving

$$
(2 \pi)^{n / 2} \int_{K} \Phi_{\alpha, \alpha}(\sigma \cdot(2 i y, 2 i v)) d \sigma=\frac{k !(n-1) !}{(k+n-1) !} \varphi_{k}(2 i y, 2 i v)
$$

whenever $|\alpha|=k$. But this is a well known fact. A representation-theoretic proof of it can be found in Ratnakumar et al. [10].

(Another way to see this is the following. The functions $\Phi_{\alpha, \alpha}(x, u)$ are eigenfunctions of the special Hermite operator $L$ with eigenvalue $2|\alpha|+n$ (see Section 1.4 in [12]). Hence $\int_{K} \Phi_{\alpha, \alpha}(\sigma \cdot(x, u)) d \sigma$ is a radial eigenfunction of the same operator. But any bounded radial eigenfunction with eigenvalue $2 k+n$ is a constant multiple of $\varphi_{k}(x, u)$. This proves that

$$
(2 \pi)^{n / 2} \int_{K} \Phi_{\alpha, \alpha}(\sigma \cdot(x, u)) d \sigma=\frac{k !(n-1) !}{(k+n-1) !} \varphi_{k}(x, u)
$$

and hence they are the same on $\mathbb{C}^{n} \times \mathbb{C}^{n}$ as well.) 
We now turn our attention to the formula for the action of $D$. The idea is to expand the operator-valued function $\pi(k(\theta) .(z, w))$ into a Fourier series. Defining

$$
\pi_{m}(z, w) F(\xi)=(2 \pi)^{-n} \int_{D} \pi(k(\theta) \cdot(z, w)) F(\xi) e^{-i m \cdot \theta} d \theta
$$

we have the expansion

$$
\pi(k(\theta) \cdot(z, w)) F(\xi)=\sum_{m \in \mathbb{Z}^{n}} \pi_{m}(z, w) F(\xi) e^{i m \cdot \theta} .
$$

By the orthogonality of the Fourier series we obtain

$$
(2 \pi)^{-n} \int_{\mathbb{R}^{n}} \int_{D}|\pi(k(\theta) .(z, w)) F(\xi)|^{2} d \theta d \xi=\sum_{m \in \mathbb{Z}^{n}} \int_{\mathbb{R}^{n}}\left|\pi_{m}(z, w) F(\xi)\right|^{2} d \xi .
$$

In calculating the $L^{2}$ norm of $\pi_{m}(z, w) F$ we make use of another property of special Hermite functions, namely that $\Phi_{\alpha, \beta}(x, u)$ is $(\beta-\alpha)$-homogeneous. By this we mean that

$$
\Phi_{\alpha, \beta}(k(\theta) \cdot(x, u))=e^{i(\beta-\alpha) \cdot \theta} \Phi_{\alpha, \beta}(x, u) .
$$

A proof of this can be found in [12, Proposition 1.4.2].

Expanding $f$ in terms of the Hermite basis we see that

$$
\pi_{m}(z, w) F=\sum_{\alpha, \beta}\left(f, \Phi_{\alpha}\right)\left(\pi_{m}(z, w) \Phi_{\alpha}, \Phi_{\beta}\right) \Phi_{\beta} .
$$

But

$$
\left(\pi_{m}(x, u) \Phi_{\alpha}, \Phi_{\beta}\right)=(2 \pi)^{-n / 2} \int_{D} \Phi_{\alpha, \beta}(k(\theta) \cdot(x, u)) e^{-i m \cdot \theta} d \theta=0
$$

unless $\beta=\alpha+m$ due to the homogeneity properties of the special Hermite functions. Therefore, the expansion of $\pi_{m}(z, w) F$ reduces to

$$
\pi_{m}(z, w) F=(2 \pi)^{n / 2} \sum_{\alpha \in \mathbb{N}^{n}}\left(f, \Phi_{\alpha}\right) \Phi_{\alpha, \alpha+m}(z, w) \Phi_{\alpha+m} .
$$

This leads us to

$$
\left\|\pi_{m}(z, w) F\right\|_{2}^{2}=(2 \pi)^{n} \sum_{\alpha \in \mathbb{N}^{n}}\left|\left(f, \Phi_{\alpha}\right)\right|^{2}\left|\Phi_{\alpha, \alpha+m}(z, w)\right|^{2} .
$$

Thus we have proved

$$
\begin{aligned}
(2 \pi)^{-n} \int_{\mathbb{R}^{n}} \int_{D}|\pi(k(\theta) .(z, w)) F(\xi)|^{2} d \theta d \xi & \\
& =(2 \pi)^{n} \sum_{m \in \mathbb{Z}^{n}} \sum_{\alpha \in \mathbb{N}^{n}}\left|\left(f, \Phi_{\alpha}\right)\right|^{2}\left|\Phi_{\alpha, \alpha+m}(z, w)\right|^{2} .
\end{aligned}
$$

This proves our claim since the sum over $m \in \mathbb{Z}^{n}$ is precisely

$$
(2 \pi)^{-n / 2} e^{u \cdot y-v \cdot x} \Phi_{\alpha, \alpha}(2 i y, 2 i v)
$$

in view of Lemma 2.2. Hence the proof of the theorem is complete. 
The above theorem has a natural converse which we state and prove now. Together they prove Theorem 1.1 stated in the introduction. In the proof of the above theorem the hypotheses on the Hermite projections of $f$ are used twice. First we used the estimates to conclude that $f$ has an entire extension to $\mathbb{C}^{n}$. Then we used them to show that the sum and the integral appearing in the above theorem are finite. In the next theorem we begin with an entire function for which the integral is finite and obtain the estimates on the projections.

TheOREM 3.2. Let $F$ be an entire function on $\mathbb{R}^{n}$ for which the integral

$$
\int_{\mathbb{R}^{n}} \int_{K}|\pi(\sigma \cdot(z, w)) F(\xi)|^{2} d \sigma d \xi
$$

is finite for all $z, w \in \mathbb{C}^{n}$. Then $\left\|P_{k} f\right\|_{2} \leq C_{t} e^{-2 k^{1 / 2} t}$ for all $t>0$.

Proof. We proceed as in the proof of the previous theorem. Since $F$ is holomorphic, $\pi(z, w) F$ makes sense. As before, for almost every $\sigma \in U(n)$ we have

$$
\int_{\mathbb{R}^{n}} \int_{D}|\pi(k(\theta) \sigma \cdot(z, w)) F(\xi)|^{2} d \theta d \xi<\infty .
$$

Expanding the operator $\pi(k(\theta) .(z, w))$ into a Fourier series and proceeding exactly as in the previous theorem, and noting that at each stage the resulting sums are finite, we get Gutzmer's formula, namely the integral in the theorem is equal to

$$
e^{u \cdot y-v \cdot x} \sum_{k=0}^{\infty} \frac{k !(n-1) !}{(k+n-1) !} \varphi_{k}(2 i y, 2 i v)\left\|P_{k} f\right\|_{2}^{2}
$$

and hence the sum is finite. Now Perron's formula for Laguerre functions on the negative real axis also gives lower bounds. That is, the Laguerre functions $\varphi_{k}(2 i y, 2 i v)$ behave like $e^{2 k^{1 / 2}\left(|y|^{2}+|v|^{2}\right)^{1 / 2}}$. In view of this we immediately get the decay estimates on the projections $P_{k} f$.

4. Some consequences. In this section we deduce some interesting consequences of our Gutzmer's formula. First we obtain the characterisation of the image of $L^{2}\left(\mathbb{R}^{n}\right)$ under the Hermite semigroup mentioned in Corollary 1.2. As pointed out earlier, the result is not new but we give a different proof.

Consider the heat kernel $p_{t}(y, v)$ associated to the special Hermite operator, which is explicitly given by

$$
p_{t}(y, v)=(2 \pi)^{-n}(\sinh (t))^{-n} e^{-\frac{1}{4} \operatorname{coth}(t)\left(|y|^{2}+|v|^{2}\right)} .
$$


Let $F=e^{-t H} f, f \in L^{2}\left(\mathbb{R}^{n}\right)$, and look at the integral

$$
\int_{\mathbb{R}^{n}}\left(\int_{\mathbb{R}^{2 n}}|\pi(i y, i v) F(\xi)|^{2} p_{2 t}(2 y, 2 v) d y d v\right) d \xi
$$

Since the function $p_{t}(y, v)$ and the Lebesgue measure $d y d v$ are both invariant under the action of the group $K$ we can rewrite the above integral as

$$
\int_{\mathbb{R}^{2 n}}\left(\int_{\mathbb{R}^{n}} \int_{K}|\pi(\sigma .(i y, i v)) F(\xi)|^{2} d \sigma d \xi\right) p_{2 t}(2 y, 2 v) d y d v
$$

In view of Gutzmer's formula the above reduces to

$$
\sum_{k=0}^{\infty} \frac{k !(n-1) !}{(k+n-1) !}\left(\int_{\mathbb{R}^{2 n}} \varphi_{k}(2 i y, 2 i v) p_{2 t}(2 y, 2 v) d y d v\right)\left\|P_{k} f\right\|_{2}^{2} e^{-2(2 k+n) t} .
$$

We now make use of the fact that

$$
\frac{k !(n-1) !}{(k+n-1) !} \int_{\mathbb{R}^{2 n}} \varphi_{k}(2 i y, 2 i v) p_{2 t}(2 y, 2 v) d y d v=e^{2(2 k+n) t},
$$

which we have established in [15, Lemma 6.3]. Therefore, we are led to the identity

$$
\int_{\mathbb{R}^{2 n}}\left(\int_{\mathbb{R}^{n}}|\pi(i y, i v) F(\xi)|^{2} d \xi\right) p_{2 t}(2 y, 2 v) d y d v=\sum_{k=0}^{\infty}\left\|P_{k} f\right\|_{2}^{2}=\int_{\mathbb{R}^{n}}|f(\xi)|^{2} d \xi
$$

A simple calculation shows that the above integral is equal to

$$
(2 \pi \sinh (2 t))^{-n} \int_{\mathbb{R}^{2 n}}\left(\int_{\mathbb{R}^{n}}|F(\xi+i v)|^{2} e^{-2 y \cdot \xi} e^{-\operatorname{coth}(2 t)\left(|y|^{2}+|v|^{2}\right)} d y\right) d \xi d v .
$$

Performing the integration with respect to $y$ we see that the above is nothing but

$$
\int_{\mathbb{R}^{2 n}}|F(\xi+i v)|^{2} U_{t}(\xi, v) d \xi d v
$$

This completes the proof of one implication of Corollary 1.2.

To prove the converse, suppose $F$ is entire and

$$
\int_{\mathbb{R}^{2 n}}|F(\xi+i v)|^{2} U_{t}(\xi, v) d \xi d v<\infty .
$$

Then it follows that for almost all $(y, v) \in \mathbb{R}^{2 n}$ we have

$$
\int_{\mathbb{R}^{n}} \int_{K}|\pi(\sigma(i y, i v)) F(\xi)|^{2} d \sigma d \xi<\infty .
$$

We can now proceed as in the proof of Theorem 3.2 to conclude that the projections $P_{k} F$ of $F$ satisfy $\left\|P_{k} F\right\|_{2} \leq C e^{-(2 k+n)^{1 / 2} s}$ for all $s>0$ and so 
Gutzmer's formula is valid for $F$. Hence the integrability of

$$
\int_{\mathbb{R}^{n}} \int_{K}|\pi(\sigma(i y, i v)) F(\xi)|^{2} d \sigma d \xi
$$

with respect to $p_{2 t}(2 y, 2 v) d y d v$ is equivalent to $F=e^{-t H} f$ for some $f \in$ $L^{2}\left(\mathbb{R}^{n}\right)$. This completes the proof of Corollary 1.2.

We remark that if we only assume the estimate $\left\|P_{k} f\right\|_{2} \leq C e^{-2 k^{1 / 2} t}$ for some $t>0$ (not for all $t$ as in Theorem 3.1) then the proof of Theorem 3.1 shows that $f$ can be extended as a holomorphic function to a certain tube domain $\Omega_{t}=\left\{z \in \mathbb{C}^{n}:|y|<t\right\}$ and still we have Gutzmer's formula as long as $|y|^{2}+|v|^{2}<t^{2}$. We can use Gutzmer's formula to obtain a characterisation of the image of $L^{2}\left(\mathbb{R}^{n}\right)$ under the Hermite-Poisson semigroup $e^{-t H^{1 / 2}}$ as a weighted Bergman space (the details will appear elsewhere). The work of Janssen and Eijndhoven [5] on the growth of Hermite coefficients treats similar problems in terms of pointwise estimates.

Another interesting consequence of Gutzmer's formula is the following orthogonality relations for Hermite functions on $\mathbb{C}^{n}$. Polarising Gutzmer we obtain

$$
\begin{aligned}
\int_{\mathbb{R}^{n}} \int_{K} \pi(\sigma \cdot(z, w)) F(\xi) & \overline{\pi(\sigma \cdot(z, w)) G(\xi)} d \sigma d \xi \\
& =e^{u \cdot y-v \cdot x} \sum_{k=0}^{\infty} \frac{k !(n-1) !}{(k+n-1) !} \varphi_{k}(2 i y, 2 i v)\left(P_{k} f, P_{k} g\right) .
\end{aligned}
$$

Specialising to Hermite functions we get the following result which, to our knowledge, seems to be new.

Corollary 4.1. For any $z, w \in \mathbb{C}^{n}$ and $\alpha, \beta \in \mathbb{N}^{n}$ we have

$$
\begin{aligned}
\int_{\mathbb{R}^{n}} \int_{K} \pi(\sigma \cdot(z, w)) \Phi_{\alpha}(\xi) \overline{\pi(\sigma \cdot(z, w)) \Phi_{\beta}(\xi)} d \sigma d \xi & \\
& =e^{u \cdot y-v \cdot x} \frac{k !(n-1) !}{(k+n-1) !} \varphi_{k}(2 i y, 2 i v) \delta_{\alpha, \beta} .
\end{aligned}
$$

The above shows that in the one-dimensional case the Hermite functions $h_{k}$ satisfy the following relations. The choice $z=i \eta, w=0$ gives

$$
\int_{\mathbb{R}}^{2 \pi} \int_{0}^{-2 \xi \eta \cos \theta} h_{k}(\xi+i \eta \sin \theta) \overline{h_{j}(\xi+i \eta \sin \theta)} d \theta d \xi=2 \pi L_{k}^{0}\left(-2 \eta^{2}\right) e^{\eta^{2}} \delta_{k, j} .
$$

The choice $z=\eta, w=i \eta$ leads to

$$
\int_{\mathbb{R}}^{2 \pi} \int_{0}^{2 \pi} e^{2 \xi \eta \sin \theta-\eta^{2} \cos (2 \theta)} h_{k}\left(\xi+i \eta e^{-i \theta}\right) \overline{h_{j}\left(\xi+i \eta e^{-i \theta}\right)} d \theta d \xi=2 \pi L_{k}^{0}\left(-2 \eta^{2}\right) \delta_{k, j} .
$$


Other interesting relations in higher-dimensional cases can be obtained by suitable choices of $z, w$ and also by choosing various subgroups of $K$.

\section{References}

[1] D.-W. Byun, Inversions of Hermite semigroup, Proc. Amer. Math. Soc. 118 (1993), 437-445.

[2] J. Faraut, Formule de Gutzmer pour la complexification d'un espace riemannien symétrique, Rend. Lincei Mat. Appl. 13 (2002), 233-241.

[3] - Analysis on the crown of a Riemannian symmetric space, in: Lie Groups and Symmetric Spaces, Amer. Math. Soc. Transl. 210, Amer. Math. Soc., Providence, RI, 2003, 99-110.

[4] G. B. Folland, Harmonic Analysis in Phase Space, Ann. of Math. Stud. 122, Princeton Univ. Press, Princeton, NJ, 1989.

[5] A. J. E. M. Janssen and S. J. L. van Eijndhoven, Spaces of type W, growth of Hermite coefficients, Wigner distribution, and Bargmann transform, J. Math. Anal. Appl. 152 (1990), 368-390.

[6] D. Karp, Square summability with geometric weight for classical orthogonal expansions, in: Advances in Analysis, H. G. W. Begehr et al. (eds.), World Sci., Hackensack, NJ, 2005, 407-421.

[7] B. Krötz, G. Ólafsson and R. J. Stanton, The image of the heat kernel transform on Riemannian symmetric spaces of the noncompact type, Int. Math. Res. Notices 2005, no. 22, 1307-1329.

[8] B. Krötz, S. Thangavelu and Y. Xu, The heat kernel transform for the Heisenberg group, J. Funct. Anal. 225 (2005), 301-336.

[9] M. Lassalle, Séries de Laurent des fonctions holomorphes dans la complexification d'un espace symétrique compact, Ann. Sci. École Norm. Sup. 11 (1978), 167-210.

[10] P. K. Ratnakumar, R. Rawat and S. Thangavelu, A restriction theorem for the Heisenberg motion group, Studia Math. 126 (1997), 1-12.

[11] G. Szegö, Orthogonal Polynomials, 3rd ed., Amer. Math. Soc. Colloq. Publ. 23, Providence, RI, 1967.

[12] S. Thangavelu, Harmonic Analysis on the Heisenberg Group, Progr. Math. 159, Birkhäuser, Boston, 1998.

[13] -, An Introduction to the Uncertainty Principle, Progr. Math. 217, Birkhäuser, Boston, 2004.

[14] —, Hermite and Laguerre semigroups: some recent developments, CIMPA-Venezuela Lecture Notes, 2006, Sémin. Congr., to appear.

[15] - Gutzmer's formula and Poisson integrals on the Heisenberg group, Pacific J. Math. 231 (2007), 217-238.

Department of Mathematics

Indian Institute of Science

Bangalore 560 012, India

E-mail: veluma@math.iisc.ernet.in

Received May 3, 2007

Revised version January 3, 2008 